
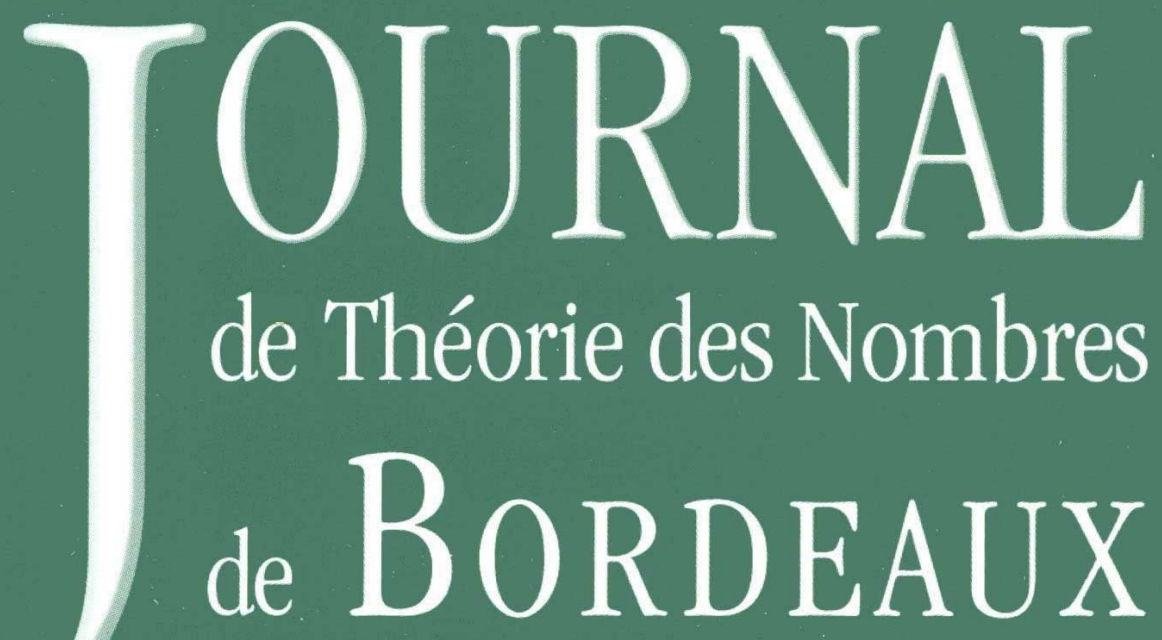

anciennement Séminaire de Théorie des Nombres de Bordeaux

Ryan DAILEDA, Jessica JOU, Robert LEMKE-OLIVER, Elizabeth ROSSOLIMO et Enrique TREVIÑO

\title{
On the counting function for the generalized Niven numbers
}

Tome 21, no 3 (2009), p. 503-515.

<http://jtnb.cedram.org/item?id=JTNB_2009__21_3_503_0>

(C) Université Bordeaux 1, 2009, tous droits réservés.

L'accès aux articles de la revue «Journal de Théorie des Nombres de Bordeaux » (http://jtnb.cedram.org/), implique l'accord avec les conditions générales d'utilisation (http://jtnb.cedram. org/legal/). Toute reproduction en tout ou partie cet article sous quelque forme que ce soit pour tout usage autre que l'utilisation à fin strictement personnelle du copiste est constitutive d'une infraction pénale. Toute copie ou impression de ce fichier doit contenir la présente mention de copyright.

\section{cedram}

Article mis en ligne dans le cadre du

Centre de diffusion des revues académiques de mathématiques

http://www.cedram.org/ 


\title{
On the counting function for the generalized Niven numbers
}

\author{
par RYAn DAILEDA, Jessica JOU, Robert LEMKE-OLIVER, \\ ELIZABETH ROSSOLIMO et ENRIQUE TREVIÑO
}

RÉSUMÉ. Étant donnés $q \geq 2$ un entier naturel et $f$ une fonction complètement q-additive à valeurs dans l'ensemble des nombres entiers relatifs, on calcule une expression asymptotique de la fonction $N_{f}$ qui à $x$ associe la cardinalité de l'ensemble

$$
\{0 \leq n<x|f(n)| n\}
$$

quand les valeurs de $f$ sont soumises à une petite restriction. Dans le cas où $f=s_{q}$, la somme des chiffres d'un nombre en base $q$, les valeurs de la function $N_{f}$ comptent les nombres q-Harshad. Donc, notre résultat généralise la formule asymptotique dans ce cas.

Abstract. Given an integer base $q \geq 2$ and a completely $q$ additive arithmetic function $f$ taking integer values, we deduce an asymptotic expression for the counting function

$$
N_{f}(x)=\#\{0 \leq n<x|f(n)| n\}
$$

under a mild restriction on the values of $f$. When $f=s_{q}$, the base $q$ sum of digits function, the integers counted by $N_{f}$ are the so-called base $q$ Niven numbers, and our result provides a generalization of the asymptotic known in that case.

\section{Introduction}

Let $q \geq 2$ be a fixed integer and let $f$ be an arbitrary complex-valued function defined on the set of nonnegative integers. We say that $f$ is completely q-additive if

$$
f\left(a q^{j}+b\right)=f(a)+f(b)
$$

for all nonnegative integers $a, b, j$ satisfying $b<q^{j}$. Given a nonnegative integer $n$, there exists a unique sequence $\left(a_{j}(n)\right)_{j \in \mathbb{N}} \in\{0,1, \ldots, q-1\}^{\mathbb{N}}$ so that

$$
n=\sum_{j=0}^{\infty} a_{j}(n) q^{j}
$$

Manuscrit reçu le 28 février 2008 .

Classification math.. 11A25, 11A63, 11K65. 
The right hand side of expression (1.1) will be called the base q expansion of $n$. Using the base $q$ expansion (1.1), we find that the function $f$ is completely $q$-additive if and only if $f(0)=0$ and

$$
f(n)=\sum_{j=1}^{\infty} f\left(a_{j}(n)\right) .
$$

It follows that a completely $q$-additive function is completely determined by its values on the set $\{0,1, \ldots, q-1\}$.

The prototypical example of a completely $q$-additive function is the base $q$ sum of digits function $s_{q}$, which is defined by

$$
s_{q}(n)=\sum_{j=0}^{\infty} a_{j}(n) .
$$

A $q$-Niven number is a positive integer $n$ that is divisible by $s_{q}(n)$. The question of the average distribution of the $q$-Niven numbers can be answered by studying the counting function

$$
N_{q}(x)=\#\left\{0 \leq n<x\left|s_{q}(n)\right| n\right\},
$$

a task which has been undertaken by several authors (see, for example, the papers of Cooper and Kennedy [1, 2, 3, 4] or De Koninck and Doyon [5]). The best known result is the asymptotic formula

$$
N_{q}(x)=\left(c_{q}+o(1)\right) \frac{x}{\log x}, \text { where } c_{q}=\frac{2 \log q}{(q-1)^{2}} \sum_{j=1}^{q-1}(j, q-1),
$$

which was proven only recently by De Koninck, Doyon and Kátai [6], and independently by Mauduit, Pomerance and Sárközy [8].

Given an arbitrary non-zero, integer-valued, completely $q$ additive function $f$, we define an $f$-Niven number to be a positive integer $n$ that is divisible by $f(n)$. In the final section of [6] it is suggested that the techniques used therein could be applied to derive an asymptotic expression for the counting function of the $f$-Niven numbers,

$$
N_{f}(x)=\#\{0 \leq n<x|f(n)| n\} .
$$

It is the goal of this paper to show that, under an additional mild restriction on $f$, this is indeed the case. Our main result is the following.

Theorem 1. Let $f$ be a non-zero, integer-valued, completely q-additive function and set

$$
\mu=\frac{1}{q} \sum_{j=0}^{q-1} f(j), \quad \sigma^{2}=\frac{1}{q} \sum_{j=0}^{q-1} f(j)^{2}-\mu^{2},
$$




$$
\begin{aligned}
& F=(f(1), f(2), \ldots, f(q-1)) \text { and } \\
& \qquad d=\operatorname{gcd}\{r f(s)-s f(r) \mid r, s \in\{1,2, \ldots, q-1\}\} .
\end{aligned}
$$

Assume $(F, q-1)=1$.

(i) If $\mu \neq 0$ then for any $\epsilon \in(0,1 / 2)$

$$
N_{f}(x)=c_{f} \frac{x}{\log x}+O\left(\frac{x}{(\log x)^{\frac{3}{2}-\epsilon}}\right)
$$

where

$$
c_{f}=\frac{\log q}{|\mu|}\left(\frac{1}{q-1} \sum_{j=1}^{q-1}(j, q-1, d)\right) .
$$

The implied constant depends only on $f$ and $\epsilon$.

(ii) If $\mu=0$ then

$$
N_{f}(x)=c_{f} \frac{x \log \log x}{(\log x)^{\frac{1}{2}}}+O\left(\frac{x}{(\log x)^{\frac{1}{2}}}\right)
$$

where

$$
c_{f}=\left(\frac{\log q}{2 \pi \sigma^{2}}\right)^{\frac{1}{2}}\left(\frac{1}{q-1} \sum_{j=1}^{q-1}(j, q-1, d)\right) .
$$

The implied constant depends only on $f$.

The hypothesis $(F, q-1)=1$ is the restriction alluded to above. It is technical in nature and will not be utilized until the end of Section 4.1. There we will see that it allows us to reduce the study of the distribution of the values of $f$ when its argument is restricted to specified congruence classes to the study of how the values themselves are distributed in congruence classes. While this hypothesis prevents Theorem 1 from being as general as one might hope, it is worth noting that it is not terribly restrictive either, especially when $q$ is large. Fixing $q \geq 3$, if we choose integer values for $f(1), f(2), \ldots, f(q-1)$ (uniformly) randomly in the interval $[-n, n]$, then $f$ is completely determined and the probability that $F=1$ is $\zeta(q-1)^{-1}+o(1)$, as $n \rightarrow \infty$. Since $\zeta(q-1)$ tends to 1 as $q$ tends to infinity, we see that for large $q$ almost all completely $q$-additive functions $f$ satisfy $(F, q-1)=1$. It would still be of interest, however, to obtain the analogue of Theorem 1 in the case that $(F, q-1)>1$.

Most of the proof of Theorem 1 is a straightforward generalization of the methods used in [6] and it is the intent of this paper to indicate where significant and perhaps non-obvious modifications to that work must be made. The first notable change is the introduction and use of the quantities $d$ and $F$. As $d=0$ and $F=1$ when $f=s_{q}$ these quantities play no role in earlier work. 
Finally, in order to shorten the presentation, we have attempted to unify the $\mu \neq 0$ and $\mu=0$ cases for as long as possible. The result is a slightly different approach in the $\mu \neq 0$ case than appears in [6]. The final steps when $\mu=0$ are entirely new.

\section{Notation}

We denote by $\mathbb{Z}, \mathbb{Z}^{+}, \mathbb{Z}_{0}^{+}, \mathbb{R}$ and $\mathbb{C}$ the sets of integers, positive integers, non-negative integers, real numbers and complex numbers, respectively. For the remainder of this paper, we fix an integer $q \geq 2$ and a non-zero completely $q$ additive function $f: \mathbb{Z}_{0}^{+} \rightarrow \mathbb{Z}$. The variable $x$ will always be assumed real and positive and $n$ will always be an integer. Following [6] we set

$$
\begin{aligned}
A(x \mid k, l, t) & =\#\{0 \leq n<x \mid n \equiv l \quad(\bmod k) \text { and } f(n)=t\} \\
a(x \mid t) & =\#\{0 \leq n<x \mid f(n)=t\}
\end{aligned}
$$

As usual, we use the notation $F(x)=O(G(x)$ ) (or $F(x) \ll G(x)$ ) to mean that there is a constant $C$ so that for all sufficiently large $x,|F(x)| \leq$ $C G(x)$. The constant $C$ and the size of $x$ are allowed to depend on $f$ (and hence on $q$ ), but on no other quantity unless specified.

\section{Preliminary Lemmas}

For real $y$ we define $\|y\|$ to be the distance from $y$ to the nearest integer.

Lemma 1. Let $M=\max _{1 \leq j \leq q-1}|f(j)|$. Let $s, k \in \mathbb{Z}^{+}$with $(s, k)=1$ and suppose that there is a pair $j_{1}, j_{2} \in\{0,1, \ldots, q-1\}$ so that $k \nmid j_{1} f\left(j_{2}\right)-$ $j_{2} f\left(j_{1}\right)$. Then for any $\xi \in \mathbb{R}$

$$
\max _{0 \leq j \leq q-1}\left\|f(j) \xi+\frac{j s}{k}\right\| \geq \frac{1}{2 M k} .
$$

Proof. We first claim that the maximum in question is at least positive. To see this we argue by contradiction and assume that $\|f(j) \xi+j s / k\|=0$ for $0 \leq j \leq q-1$. Then $f(j) \xi+j s / k=n_{j} \in \mathbb{Z}$ for each $j$. Solving for $\xi$ and equating the resulting expressions, we find that if $f\left(j_{1}\right), f\left(j_{2}\right) \neq 0$ then

$$
k\left(n_{j_{1}} f\left(j_{2}\right)-n_{j_{2}} f\left(j_{1}\right)\right)=s\left(j_{1} f\left(j_{2}\right)-j_{2} f\left(j_{1}\right)\right) .
$$

Since $(s, k)=1$, this implies that $k \mid j_{1} f\left(j_{2}\right)-j_{2} f\left(j_{1}\right)$, which is excluded by assumption. Since this same condition is trivially verified if $f\left(j_{1}\right)=0$ or $f\left(j_{2}\right)=0$, we have a contradiction in any case. 
To prove the lemma we again argue by contradiction. Let us remark that for any $k>0$

$$
\begin{aligned}
\frac{1}{k} & \leq\left\|\frac{\left(j_{1} f\left(j_{2}\right)-j_{2} f\left(j_{1}\right)\right) s}{k}\right\| \\
& =\left\|\left(f\left(j_{1}\right) f\left(j_{2}\right) \xi+\frac{j_{1} f\left(j_{2}\right) s}{k}\right)-\left(f\left(j_{1}\right) f\left(j_{2}\right) \xi+\frac{j_{2} f\left(j_{1}\right) s}{k}\right)\right\| \\
& \leq\left\|f\left(j_{2}\right)\left(f\left(j_{1}\right) \xi+\frac{j_{1} s}{k}\right)\right\|+\left\|f\left(j_{1}\right)\left(f\left(j_{2}\right) \xi+\frac{j_{2} s}{k}\right)\right\| .
\end{aligned}
$$

If we assume now that (3.1) is not verified, then

$$
\begin{aligned}
\left|f\left(j_{1}\right)\right| & \leq M \\
& <\frac{1}{2 k \max _{0 \leq j \leq q-1}\|f(j) \xi+j s / k\|} \\
& \leq \frac{1}{2\left\|f\left(j_{2}\right) \xi+j_{2} s / k\right\|}
\end{aligned}
$$

and similarly

$$
\left|f\left(j_{2}\right)\right|<\frac{1}{2\left\|f\left(j_{1}\right) \xi+j_{1} s / k\right\|} .
$$

Therefore, by properties of $\|\cdot\|$,

$$
\begin{aligned}
\frac{1}{k} & \leq\left|f\left(j_{2}\right)\right| \cdot\left\|f\left(j_{1}\right) \xi+\frac{j_{1} s}{k}\right\|+\left|f\left(j_{1}\right)\right| \cdot\left\|f\left(j_{2}\right) \xi+\frac{j_{2} s}{k}\right\| \\
& \leq 2 M \max _{0 \leq j \leq q-1}\left\|f(j) \xi+\frac{j s}{k}\right\| \\
& <2 M \frac{1}{2 M k} \\
& =\frac{1}{k}
\end{aligned}
$$

which is a contradiction.

It is proven in [7] that if $z_{1}, z_{2}, \ldots, z_{q-1} \in \mathbb{C}$ satisfy $\left|z_{j}\right| \leq 1$ for $j=$ $1,2, \ldots, q-1$. then

$$
\left|\frac{1}{q}\left(1+\sum_{j=1}^{q-1} z_{j}\right)\right| \leq 1-\frac{1}{2 q} \max _{1 \leq j \leq q-1}\left(1-\operatorname{Re} z_{j}\right) .
$$

Since for real $y$ we have $\|y\|^{2} \ll 1-\cos (2 \pi y)$ and $1+y \leq e^{y}$, the next lemma is an immediate consequence. 
Lemma 2. Let $e(y)=e^{2 \pi i y}$. There is a positive constant $c_{1}=c_{1}(f)$ so that for any $\xi, r \in \mathbb{R}$

$$
\left|\frac{1}{q} \sum_{j=0}^{q-1} e(f(j) \xi+r j)\right| \leq \exp \left(-c_{1} \max _{1 \leq j \leq q-1}\|f(j) \xi+r j\|^{2}\right) .
$$

\section{The distribution of the values of $f$}

4.1. Argument restricted to a congruence class. Let $k \in \mathbb{Z}^{+}, l \in \mathbb{Z}_{0}^{+}$ and $t \in \mathbb{Z}$. Our first goal in this section is to relate $A(x \mid k, l, t)$ to the function $a(x \mid t)$ through a series of reductions on the modulus $k$. The first three reductions are proven exactly as in [6], substituting our Lemma 2 for their Lemma 3. We state them for the convenience of the reader.

Reduction 1. Write $k=k_{1} k_{2}$ where $k_{1}$ is the largest divisor of $k$ so that $\left(k_{1}, q\right)=1$. Then the primes dividing $k_{2}$ also divide $q$ and we let $h$ be the smallest positive integer so that $k_{2}$ divides $q^{h}$. Since $k$ divides $k_{1} q^{h}$, the congruence class $l(\bmod k)$ is the union of classes $l^{(j)}\left(\bmod k_{1} q^{h}\right)$, $j=1, \ldots, q^{h} / k_{2}$. For each $j$ write

$$
l^{(j)}=l_{1}^{(j)}+q^{h} l_{2}^{(j)}
$$

where $0 \leq l_{1}^{(j)}<q^{h}$. Then

$$
A(x \mid k, l, t)=\sum_{j=1}^{q^{h} / k_{2}} A\left(\frac{x-l_{1}^{(j)}}{q^{h}} \mid k_{1}, l_{2}^{(j)}, t-f\left(l_{1}^{(j)}\right)\right) .
$$

Since $\left(k_{1}, q\right)=1$, we are led to the next reduction.

Reduction 2. Suppose that $(k, q)=1$. Let $k=k_{1} k_{2}$ where $k_{1}$ is the largest divisor of $k$ so that $\left(k_{1}, q-1\right)=1$. Then there is a positive constant $c_{3}=c_{3}(f)$ so that

$$
A(x \mid k, l, t)=\frac{1}{k_{1}} A\left(x \mid k_{2}, l, t\right)+O\left(x^{1-\frac{c_{3}}{\log 2 k}}\right) .
$$

Before moving to the next reduction, we note that the primes dividing $k_{2}$ must also divide $q-1$.

Reduction 3. Suppose that the prime divisors of $k$ also divide $q-1$. Then there is a positive constant $c_{4}=c_{4}(f)$ so that

$$
A(x \mid k, l, t)=\frac{(k, q-1)}{k} A(x \mid(k, q-1), l, t)+O\left(x^{1-\frac{c_{4}}{\log 2 k}}\right) .
$$

We have therefore reduced to the case in which the modulus is a divisor of $q-1$.

Reduction 4. We now come to the first new reduction. The proof closely follows that of Reductions 2 and 3 of [6], substituting our Lemma 1 for their Lemma 1 and our Lemma 2 for their Lemma 3. We therefore choose to omit it. Suppose that $k \mid q-1$ and let $d$ denote the greatest common 
divisor of $j_{1} f\left(j_{2}\right)-j_{2} f\left(j_{1}\right)$ for $j_{1}, j_{2} \in\{0,1, \ldots, q-1\}$. Then there is a positive constant $c_{5}=c_{5}(f)$ so that

$$
A(x \mid k, l, t)=\frac{(k, d)}{k} A(x \mid(k, d), l, t)+O\left(x^{1-c_{5}}\right) .
$$

We note that in the case $f=s_{q}$ we have $d=0$, making this reduction unnecessary. As such, it has no analogue in [6].

Reduction 5. Suppose that $k$ divides $(q-1, d)$. Then $q^{j} \equiv 1(\bmod k)$ for all $j \geq 1$ and $j_{1} f\left(j_{2}\right) \equiv j_{2} f\left(j_{1}\right)(\bmod k)$ for all $0 \leq j_{1}, j_{2} \leq q-1$. From this and the complete $q$-additivity of $f$ it follows that $m f(n) \equiv n f(m)$ $(\bmod k)$ for all $m, n \in \mathbb{Z}_{0}^{+}$. Let $F=(f(1), f(2), \ldots, f(q-1))$ and suppose further that $(F, q-1)=1$. Since the values of $f$ are linear combinations of $f(1), f(2), \ldots, f(q-1)$ with nonnegative integer coefficients, the condition $(F, q-1)=1$ implies that we can find an $m \in \mathbb{Z}^{+}$so that $(f(m), q-1)=1$. Therefore if $n \in \mathbb{Z}_{0}^{+}$satisfies $f(n)=t$ we have $m t=m f(n) \equiv n f(m)$ $(\bmod k)$ and it follows that

$$
A(x \mid k, l, t)= \begin{cases}a(x \mid t) & \text { if } m t \equiv l f(m) \quad(\bmod k), \\ 0 & \text { otherwise }\end{cases}
$$

This is the analogue of the final reduction in section 4.5 of [6].

4.2. Unrestricted argument. We now turn to the distribution of the values of $f$ when its argument is free to take on any value. We let $\mu$ and $\sigma$ denote the mean and standard deviation of $f$ on the set $D=\{0,1, \ldots, q-1\}$ of base $q$ digits. That is,

$$
\mu=\frac{1}{q} \sum_{j=0}^{q-1} f(j), \sigma^{2}=\frac{1}{q} \sum_{j=0}^{q-1} f(j)^{2}-\mu^{2} .
$$

We also set

$$
N_{x}=\left\lfloor\frac{\log x}{\log q}\right\rfloor
$$

where $\lfloor y\rfloor$ is the greatest integer not exceeding $y$.

Following [1], we view $D$ as a probability space in which each element is assigned a measure of $1 / q$. We use the digits of the base $q$ expansion (1.1) to identify the set of nonnegative integers $n$ strictly less than $q^{N}\left(N \in \mathbb{Z}^{+}\right)$ with the product space $D^{N}$. In this way $f$ can be viewed as the random variable on $D^{N}$ obtained by summing together $N$ independent copies of $f$ acting on $D$ alone. In this setting, if we apply Theorem 15, Chapter III of [9] we immediately obtain the next lemma.

Lemma 3. Let $F: D \rightarrow \mathbb{R}$ have average value 0 . If $F$ is extended to a completely $q$-additive function on $\mathbb{Z}_{0}^{+}$, then there is a constant $C>0$, depending only on $q$ and $F$, so that for $N \in \mathbb{Z}^{+}$we have

$$
\#\left\{0 \leq n<q^{N} \mid F(n) \geq k\right\} \leq q^{N} e^{-C k^{2} / N}
$$


and

$$
\#\left\{0 \leq n<q^{N} \mid F(n) \leq-k\right\} \leq q^{N} e^{-C k^{2} / N}
$$

for all $0 \leq k \leq N / C$.

Proposition 1. Let $\epsilon \in(0,1 / 2)$ and set $I=\left[\mu N_{x}-N_{x}^{1 / 2+\epsilon}, \mu N_{x}+N_{x}^{1 / 2+\epsilon}\right]$. Then

$$
\#\{0 \leq n<x \mid f(n) \notin I\} \ll \frac{x}{(\log x)^{2}}
$$

the implied constant depending only on $f$ and $\epsilon$.

Proof. For $C>0$, as $N \in \mathbb{Z}^{+}$tends to infinity eventually $0 \leq N^{1 / 2+\epsilon} \pm \mu \leq$ $(N+1) / C$, so that Lemma 3 applied to the function $F=f-\mu$ gives

$$
\#\left\{0 \leq n<q^{N+1} \mid f(n)-\mu N \geq N^{1 / 2+\epsilon}\right\} \ll q^{N} e^{-c_{1} N^{2 \epsilon}}
$$

and

$$
\#\left\{0 \leq n<q^{N+1} \mid f(n)-\mu N \leq-N^{1 / 2+\epsilon}\right\} \ll q^{N} e^{-c_{2} N^{2 \epsilon}}
$$

for some positive constants $c_{1}$ and $c_{2}$. Setting $c=\min \left\{c_{1}, c_{2}\right\}$ these inequalities together give

$$
\#\left\{0 \leq x<q^{N+1}|| f(n)-\mu N \mid \geq N^{1 / 2+\epsilon}\right\} \ll q^{N} e^{-c N^{2 \epsilon}} .
$$

The stated result now follows since

$$
\begin{aligned}
\#\{0 \leq n<x \mid f(n) \notin I\} & \leq \#\left\{0 \leq n<q^{N_{x}+1}|| f(n)-\mu N_{x} \mid \geq N_{x}^{1 / 2+\epsilon}\right\} \\
& \ll q^{N_{x}} e^{-c N_{x}^{2 \epsilon}} \\
& \ll \frac{x}{(\log x)^{2}} .
\end{aligned}
$$

We remark that if $\epsilon>0$, Lemma 4 of [8], when translated into our present notation, immediately implies that

$$
\#\left\{0 \leq n<x \mid s_{q}(n) \notin I\right\} \ll \max \left\{x \exp \left(-\frac{6}{q^{2}-1} N_{x}^{2 \epsilon}\right), x^{1-\frac{c}{\log \log x}}\right\}
$$

for some $c>0$. Since the right hand side of $(4.2)$ is $O\left(\frac{x}{(\log x)^{2}}\right)$, this provides a proof of Proposition 1 in the case $f=s_{q}$. In fact, if in the proof of Proposition 1 we replace $N_{x}^{1 / 2+\epsilon}$ by $N_{x}^{1 / 2} \lambda_{x}$, where $\lambda_{x}=o\left(N_{x}^{1 / 2}\right)$, we obtain a more general result that is very similar to Lemma 4 of [8].

As observed in [6] in the case $f=s_{q}$, Theorem 6, Chapter VII of [9] can be used to obtain the following result. 
Proposition 2. If $(f(1), f(2), \ldots, f(q-1))=1$ then uniformly for $t \in \mathbb{Z}$ we have

$$
a(x \mid t)=\frac{x}{\sigma \sqrt{N_{x}}} \varphi\left(\frac{t-\mu N_{x}}{\sigma \sqrt{N_{x}}}\right)+O\left(\frac{x}{N_{x}}\right)
$$

where $\varphi(y)=(2 \pi)^{-1 / 2} e^{-y^{2} / 2}$. The implied constant depends only on $f$.

Corollary 1. If $F=(f(1), f(2), \ldots, f(q-1))$ then

$$
a(x \mid t)=\frac{F x}{\sigma \sqrt{N_{x}}} \varphi\left(\frac{t-\mu N_{x}}{\sigma \sqrt{N_{x}}}\right)+O\left(\frac{x}{N_{x}}\right)
$$

when $t \equiv 0(\bmod F)$ and $a(x \mid t)=0$ otherwise.

Proof. Apply the proposition to the function $g=f / F$.

\section{Proof of Theorem 1}

It suffices to consider the case in which $\mu \geq 0$, since $N_{f}(x)=N_{-f}(x)$. Fixing $\epsilon \in(0,1 / 2)$, according to Proposition 1 we have

$$
N_{f}(x)=\sum_{\substack{t \in I \\ t \equiv 0}} A(x \| t \mid, 0, t)+O\left(\frac{x}{(\bmod F)}\right) .
$$

As in Section 5 of [6], one can show that Reductions 1 through 5 together with the corollary to Proposition 2 yield

$$
A(x|| t \mid, 0, t)=\frac{(t, q-1, d)}{|t|} a(x \mid t)+O\left(\frac{x \log N_{x}}{t N_{x}}\right)
$$

uniformly for (nonzero) $t \in I$. Since $(t, q-1, d)$ depends only on the residue of $t(\bmod q-1)$, substitution of (5.2) into (5.1) yields

$$
N_{f}(x)=\sum_{j=1}^{q-1}(j, q-1, d) \sum_{\substack{t \in I \\ t \neq 0 \\ t \equiv 0 \\ t \equiv j}} \frac{a(x \mid t)}{|t|}+O\left(\frac{x \log N_{x}}{N_{x}} E_{1}(x)\right)
$$

where

$$
E_{1}(x)= \begin{cases}N_{x}^{\epsilon-1 / 2} & \text { if } \mu>0 \\ \log N_{x} & \text { if } \mu=0\end{cases}
$$

Lemma 4. For $j=1,2, \ldots, q-1$ we have

$$
\sum_{\substack{t \in I \\ t \neq 0 \\ t \equiv 0 \\ t \equiv j}} \frac{a(x \mid t)}{|t|}=\frac{1}{q-1} \sum_{\substack{t \in I \\ t \neq 0 \\ t \neq 0}} \frac{a(x \mid t)}{|t|}+O\left(\frac{x}{\bmod _{x}} E_{2}(x)\right)
$$

where

$$
E_{2}(x)= \begin{cases}N_{x}^{\epsilon-1 / 2} & \text { if } \mu>0 \\ N_{x}^{1 / 2} & \text { if } \mu=0\end{cases}
$$


Proof. Since we have assumed $(F, q-1)=1$, for each $j=1,2, \ldots, q-1$ there is a unique $b_{j}(\bmod F(q-1))$ so that the two simultaneous congruences $t \equiv$ $0(\bmod F)$ and $t \equiv j(\bmod q-1)$ are equivalent to the single congruence $t \equiv b_{j}(\bmod F(q-1))$. The corollary to Proposition 2 implies that for $t, k \equiv 0(\bmod F)$ we have

$$
\frac{a(x \mid t+k)}{|t+k|}=\frac{a(x \mid t)}{|t|}+O\left(\frac{x}{|t| \log x}+\frac{x}{t^{2}(\log x)^{1 / 2}}\right)
$$

when $t+k \neq 0$, the implied constant depending only on $f$ and $k$.

When $\mu>0$ we therefore have

$$
\begin{aligned}
\sum_{\substack{t \in I \\
t \neq 0 \\
t \equiv 0 \\
(\bmod F)}} \frac{a(x \mid t)}{|t|} & =\sum_{\substack{t \in I \\
(\bmod q-1)}} \frac{a(x \mid t)}{t} \\
& =\frac{1}{q-1} \sum_{\substack{t \in I \\
(\bmod F(q-1))}} \sum_{k=0}^{q-2} \frac{a(x \mid t+k F)}{t+k F} \\
& +O\left(\frac{x}{N_{x}^{3 / 2-\epsilon}}\right) \\
& =\frac{1}{q-1} \sum_{s \in I} \frac{a(x \mid s)}{s}+O\left(\sum_{s \in J} \frac{a(x \mid s)}{s}\right) \\
& +O\left(\frac{x}{N_{x}^{3 / 2-\epsilon}}\right),
\end{aligned}
$$

where $J$ is the set of integers with distance at most $q F$ from the endpoints of $I$. Since $a(x \mid s) \ll x / N_{x}^{1 / 2}$, the sum over $J$ contributes no more than the second error term, proving the lemma in this case.

When $\mu=0$ we may carry through the same analysis, being careful in the second step to omit from the summation the single value of $t$ for which $t+k F=0$. This introduces an error of size $O\left(x / N_{x}^{1 / 2}\right)$, which is consistent with the statement of the lemma. 
Combining Lemma 4 with equation (5.3) we can now complete the proof. When $\mu>0$ we have

$$
\begin{aligned}
N_{f}(x) & =\left(\frac{1}{q-1} \sum_{j=1}^{q-1}(j, q-1, d)\right) \sum_{\substack{t \in I \\
t \equiv 0}} \frac{a(x \mid t)}{t}+O\left(\frac{x \log N_{x}}{N_{x}^{3 / 2-\epsilon}}\right) \\
& =\frac{1}{\mu N_{x}}\left(\frac{1}{q-1} \sum_{j=1}^{q-1}(j, q-1, d)\right) \sum_{\substack{t \in 0 \\
t \equiv 0}} a(x \mid t) \\
& +O\left(\frac{x \log \log x)}{(\log x)^{3 / 2-\epsilon}}\right) \\
& =\frac{\log q}{\mu \log x}\left(\frac{1}{q-1} \sum_{j=1}^{q-1}(j, q-1, d)\right) \sum_{\substack{t \in 0 \\
t(\bmod F)}} a(x \mid t) \\
& +O\left(\frac{x \log \log x}{(\log x)^{3 / 2-\epsilon}}\right) \\
& =\frac{\log q}{\mu}\left(\frac{1}{q-1} \sum_{j=1}^{q-1}(j, q-1, d)\right) \frac{x}{\log x}+O\left(\frac{x \log \log x}{(\log x)^{3 / 2-\epsilon}}\right),
\end{aligned}
$$

where in the final line we have used Proposition 1 to replace $\sum_{t \in I} a(x \mid t)$ with $x+O\left(x /(\log x)^{2}\right)$. Since $\epsilon \in(0,1 / 2)$ was arbitrary, we may discard the $\log \log x$ term in the error, giving the stated result.

When $\mu=0$ we have

$$
N_{f}(x)=\left(\frac{1}{q-1} \sum_{j=1}^{q-1}(j, q-1, d)\right) \sum_{\substack{t \in I \\ t \neq 0 \\ t \equiv 0}} \frac{a(x \mid t)}{|t|}+O\left(\frac{x}{N_{x}^{1 / 2}}\right)
$$

Set $\epsilon=1 / 4$ for convenience. Writing $t=F s$ and using the corollary to Proposition 2 we obtain

$$
\sum_{\substack{t \in I \\ t \neq 0 \\(\bmod F)}} \frac{a(x \mid t)}{|t|}=\frac{2 x}{\sigma \sqrt{N_{x}}} \sum_{1 \leq s \leq N_{x}^{3 / 4} / F} \frac{1}{s} \varphi\left(\frac{s F}{\sigma \sqrt{N_{x}}}\right)+O\left(\frac{x \log N_{x}}{N_{x}}\right) .
$$


Now

$$
\begin{aligned}
\sum_{1 \leq s \leq N_{x}^{3 / 4} / F} \frac{1}{s} \varphi\left(\frac{s F}{\sigma \sqrt{N_{x}}}\right) & =\int_{1}^{N_{x}^{3 / 4} / F} \frac{1}{s} \varphi\left(\frac{s F}{\sigma \sqrt{N_{x}}}\right) d s+O(1) \\
& =\frac{1}{\sqrt{2 \pi}} \int_{F / \sigma \sqrt{2 N_{x}}}^{N_{x}^{1 / 4} / \sigma \sqrt{2}} \frac{e^{-u^{2}}}{u} d u+O(1) \\
& =\frac{1}{\sqrt{2 \pi}} \int_{F / \sigma \sqrt{2 N_{x}}}^{1} \frac{e^{-u^{2}}}{u} d u+O(1) \\
& =\frac{1}{\sqrt{2 \pi}} \int_{F / \sigma \sqrt{2 N_{x}}}^{1} \frac{1}{u} d u+O(1) \\
& =\frac{1}{2 \sqrt{2 \pi}} \log N_{x}+O(1) \\
& =\frac{1}{2 \sqrt{2 \pi}} \log \log x+O(1) .
\end{aligned}
$$

Returning to equations (5.5) and (5.6) we find that

$$
\begin{aligned}
N_{f}(x) & =\frac{1}{\left(2 \pi \sigma^{2}\right)^{1 / 2}}\left(\frac{1}{q-1} \sum_{j=1}^{q-1}(j, q-1, d)\right) \frac{x \log \log x}{N_{x}^{1 / 2}}+O\left(\frac{x}{N_{x}^{1 / 2}}\right) \\
& =\left(\frac{\log q}{2 \pi \sigma^{2}}\right)^{1 / 2}\left(\frac{1}{q-1} \sum_{j=1}^{q-1}(j, q-1, d)\right) \frac{x \log \log x}{(\log x)^{1 / 2}} \\
& +O\left(\frac{x}{(\log x)^{1 / 2}}\right)
\end{aligned}
$$

which concludes the proof.

\section{References}

[1] C. N. Cooper, R. E. Kennedy, On the natural density of the Niven numbers. College Math. J. 15 (1984), 309-312.

[2] C. N. Cooper, R. E. Kennedy, On an asymptotic formula for the Niven numbers. Internat. J. Math. Sci. 8 (1985), 537-543.

[3] C. N. Cooper, R. E. Kennedy, A partial asymptotic formula for the Niven numbers. Fibonacci Quart. 26 (1988), 163-168.

[4] C. N. Cooper, R. E. Kennedy, Chebyshev's inequality and natural density. Amer. Math. Monthly 96 (1989), 118-124.

[5] J.-M. De Koninck, N. Doyon, On the number of Niven numbers up to x. Fibonacci Quart. 41 (5) (2003), 431-440.

[6] J.-M. De Koninck, N. Doyon, I. KÁtai, On the counting function for the Niven numbers. Acta Arith. 106 (3) (2003), 265-275.

[7] H. Delange, Sur les fonctions q-additives ou q-multiplicatives. Acta Arith. 21 (1972), 285298.

[8] C. Mauduit, C. Pomerance, A. SÁrközy, On the distribution in residue classes of integers with a fixed sum of digits. Ramanujan J. 9 (1-2) (2005), 45-62. 
[9] V. V. Petrov, Sums of Independent Random Variables. Ergeb. Math. Grenzgeb. 82, Springer, 1975.

Ryan DAILEDA

Mathematics Department

Trinity University

One Trinity Place

San Antonio, TX 78212-7200, USA

E-mail: rdaileda@trinity.edu

$U R L:$ http://www.trinity.edu/rdaileda

Jessica JoU

678-2 Azumi, Ichinomiya-cho

Shiso-shi, Hyogo 671-4131

Japan

E-mail: jou.jessica@gmail.com

Robert Lemke-Oliver

Deparment of Mathematics

University of Wisconsin Madison

480 Lincoln Dr

Madison, WI 53706 USA

E-mail: lemkeoli@math.wisc.edu

Elizabeth Rossolimo

Department of Mathematics and Statistics Lederle Graduate Research Tower

Box 34515

University of Massachusetts Amherst

Amherst, MA 01003-9305, USA

E-mail: erossoli@student.umass.edu

Enrique TREviÑo

Department of Mathematics

6188 Kemeny Hall

Dartmouth College

Hanover, NH 03755-3551, USA

E-mail: enrique.trevino@dartmouth.edu 